\title{
DFT Studies of Caffeic Acid Antioxidant: Molecular Orbitals and Composite Reactivity Maps Correlation with Photophysical Characteristics and Photochemical Stability
}

\author{
M. S. A. Abdel-Mottaleb \\ Nano-Photochemistry and Solarchemistry Labs, Department of Chemistry, Faculty of Science, \\ Ain Shams University, Abbassia, Cairo 11566, Egypt \\ Correspondence should be addressed to M. S. A. Abdel-Mottaleb; phochem08@photoenergy.org
}

Received 2 November 2015; Revised 15 December 2015; Accepted 28 December 2015

Academic Editor: Bhabani Mallik

Copyright (C) 2016 M. S. A. Abdel-Mottaleb. This is an open access article distributed under the Creative Commons Attribution License, which permits unrestricted use, distribution, and reproduction in any medium, provided the original work is properly cited.

This paper describes experimentally verifiable computational chemistry results of the environmentally benign caffeic acid (CA) antioxidant. Computations at density functional level (DFT) and its time dependent (TD) extension are carried out to explain results obtained experimentally in our laboratories. Emphases are on acidity constants, photodegradation, fluorescence quenching by metal ligation, and UV-Vis absorption characteristics of CA. Additionally, quantitative structure activity indices and composite maps that visualizing nucleophilicity, electrophilicity, and potential energy surface (PES map) are computed and discussed.

\section{Introduction}

Phenolic acids are a group of dietary phytochemicals (friendly chemical family) that act as antioxidants and flavorings and possess disease-preventive properties. Caffeic acid is an important member of this family and has been investigated in different international laboratories due to its importance in pharmaceutical industry and food applications as well as its ability to suppress tumor development. CA (Figure 1), 3,4-dihydroxycinnamic acid, is a phytochemical existing in some vegetables, medicinal herbs, and plants [1] and also in some beverages $[1,2]$. Coffee drinking was shown to increase the incorporation of conjugated forms of caffeic acid into LDL particles, and the oxidation-resistance of LDL was increased [3]. Moreover, CA was reported to have a wide variety of pharmacological activities including antioxidants [4], immunomodulatory, antiviral, anti-HIV [5], anticarcinogenic, and anti-inflammatory effect [6-9]. Recent research has shown that CA and some of its derivatives can act as substrate for polyphenols oxidases or peroxidases [10].

A summary of the research work performed so far using high accuracy quantum chemical methods on polyphenols has been published [11]. The different groups of polyphenols, which mostly belong to the Mediterranean food culture, such as phenolic acids, have been reviewed. The three main proposed mechanisms through which the antioxidants may play their protective role, which is the $\mathrm{H}$ atom transfer, the single electron transfer, and the metals chelation, have been analyzed and discussed in detail. The results represent further important contribution to the elucidation of the beneficial effects on health of these substances.

However, detailed investigations are still needed. In order to shed more light on different important characteristics of CA, our aim is focused on investigating some characteristics such as its photodegradation in presence of $\mathrm{TiO}_{2}$, which is a famous food additive, and the influence of some metal cations, its fluorescence quenching by metal cations, its $\mathrm{p} K_{\mathrm{a}}$ values determination, and detailed quantum chemical parameters that could be verified experimentally. Furthermore, graphical models computed by DFT method, in particular, molecular orbitals, electron density, and electrostatic potential maps will be investigated. The main purpose of this graphical model visualization is to explore structure-property relationships in depth by doing analysis of computational data. 


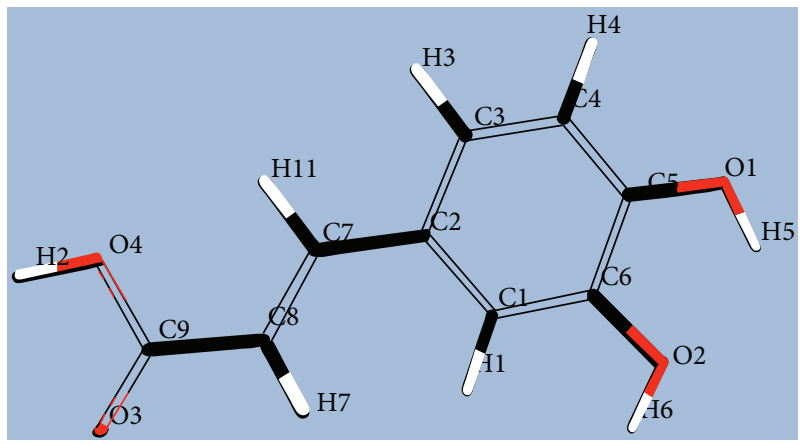

FIgURE 1: The structure and labeling of caffeic acid.

\section{Materials and Methods}

2.1. Materials. Caffeic acid (ISC company, >98\%) was used as received. Pure grade (Aldrich) solvents were used. Other chemicals such as hydrated ferric chloride and copper chloride are purchased from Riedel. Perchloric acid (Merck, 70\% purity) and sodium hydroxide having a minimum assay of 98\% from Avondale Labs were used to adjust the $\mathrm{pH}$ of the medium.

2.2. Instruments. UV-Vis absorption spectra were measured in the range of 250-800 $\mathrm{nm}$ using a double beam UNICAM spectrophotometer model $(\operatorname{He} \lambda \operatorname{ios} \alpha)$. The source of UV/Vis irradiation was Xenon discharge arc lamp with PTI model 1010 Housing. Solution pHs were measured by a calibrated Jenway ion analyzer model 3040.

2.3. General Procedure for the Photochemical Reactions. The photocatalytic reaction in a double walled quartz cell cooled by circulated water is carried out using the Xenon Arc lamp system and monitored spectrophotometrically. All the measurements were carried out at room temperature $\approx 22^{\circ} \mathrm{C}$. A suspension of $1 \mathrm{~g} /$ liter of $\mathrm{TiO}_{2}$ (Degussa P25) was prepared in all photocatalytic experiments. CA concentration was kept at $6 \times 10^{-5} \mathrm{M}$ for all measurements and reduced to $1 \times 10^{-6} \mathrm{M}$ in case of fluorescence measurements.

2.4. Theoretical Computations. Gaussian 09 package program was used for computational process [14]. Density functional theory (DFT) method, time dependent-density functional theory (TD-DFT) method, and B3LYB basis set is used in calculations.

The user-friendly Spartan'14 parallel 64-bit version (Wavefunction Inc., USA) quantum chemical package is also used within the framework of DFT and TD-DFT (with $6 \mathrm{MOs}$ taken into account in the CI). Data is visualized by Gausview 05 program (in case of Gaussian 09 computations). 12-core pro-MAC computer was used to perform the computations. The geometry optimization of CA was carried out in vacuum as implemented in the Gaussian 09 package [14]. The geometry of CA was optimized using B3LYP [15] functional with the $6-31 G^{*}$-basis set, which is a good compromise between accuracy and efficiency. Using $6-311 G^{*}$ basis set improves the
TABLE 1: TD-DFT computed UV/Vis Allowed Transitions of CA (most strong only) in gas phase.

\begin{tabular}{lccc}
\hline $\mathrm{nm}$ & Oscillator strength & \multicolumn{2}{c}{ MO component } \\
\hline 307.30 & 0.5210 & HOMO $\rightarrow$ LUMO & $91 \%$ \\
276.72 & 0.1404 & HOMO-1 $\rightarrow$ LUMO & $77 \%$ \\
213.56 & 0.2156 & HOMO-3 $\rightarrow$ LUMO & $72 \%$ \\
\hline
\end{tabular}

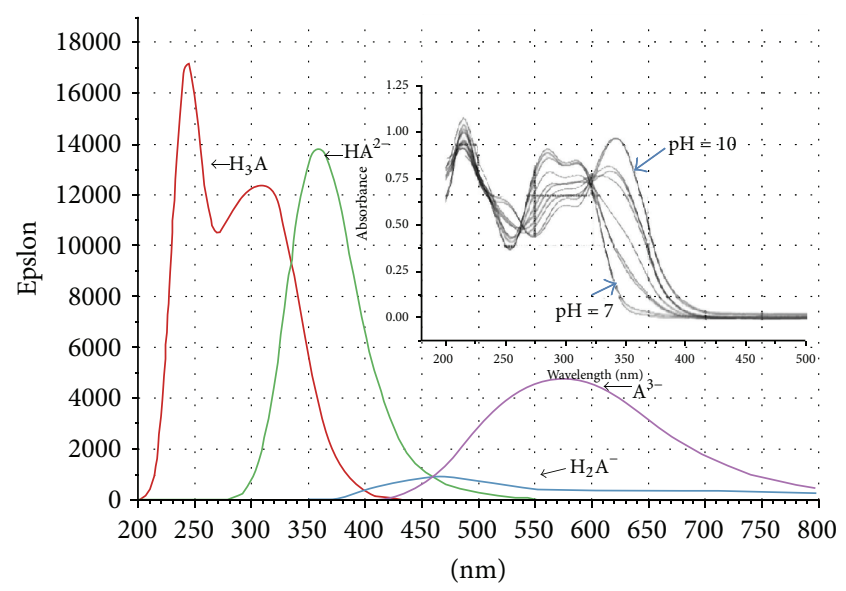

Figure 2: Computed UV/Vis spectra of $\mathrm{H}_{3} \mathrm{~A}$ [CA neutral (red)], $\mathrm{H}_{2} \mathrm{~A}^{-}$[CA Anion (cyan)], $\mathrm{HA}^{2-}$ [CA Di-anion (green)], and $\mathrm{A}^{3-}$ [CA Tri-anion (purple)] in aqueous phase. The inset shows experimentally measured absorption spectra of CA $\left(0.6 \times 10^{-4} \mathrm{M}\right)$ in media of different $\mathrm{pHs}$.

UV-Vis absorption spectral parameters. Molecular surfaces are calculated using B3LYP with $6-311 G^{*}$ basis set. First excited state $\left(\mathrm{S}_{1}\right)$ calculations are obtained at Configuration Interaction Singles (CIS) level with similar basis sets (6$\left.311 \mathrm{G}^{*}\right)$.

DataGraph 3.2 (Visual Data Tools, Inc., US) software was used for data handling and Graphical representation of the TD-DFT UV-Vis electronic spectra.

The UV/Vis spectrum was also studied in gas phase. Solvation energies were calculated using Spartan 14.

\section{Results and Discussion}

3.1. Absorption Spectrum of Caffeic Acid (CA). The absorption spectrum of CA depicted in the inset of Figure 2 shows 3 bands at around $315 \mathrm{~nm}, 278 \mathrm{~nm}$, and $217 \mathrm{~nm}$ in slightly acidic medium ( $\mathrm{pH}$ 5.9). Theoretically calculated electronic transitions as well as its intensities and MOs involved are given in Table 1 and Figure 3. The most important transition (at $307.30 \mathrm{~nm}$ and $f=0.521$ ) is of charge transfer character that originated mostly from HOMO mainly localized on the catechol moiety to the lowest unoccupied MO (LUMO) mainly spreading over the rest of the molecular fragment containing the carboxylic group. The gas phase UV/Vis transitions and the calculated spectrum in aqueous medium (Table 1 and Figure 2) are in good agreement with the experimentally measured one shown as inset in Figure 2. 


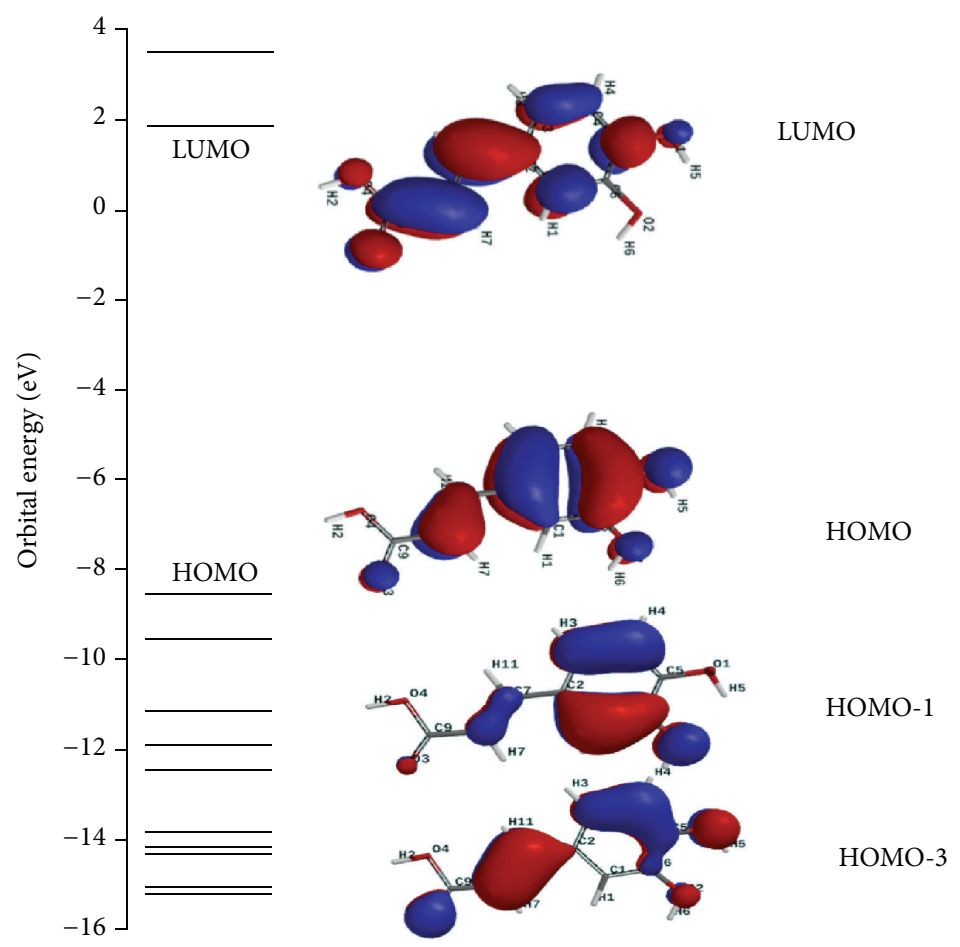

FIgURE 3: MOs involved in the UV/Vis electronic transitions of Table 1.

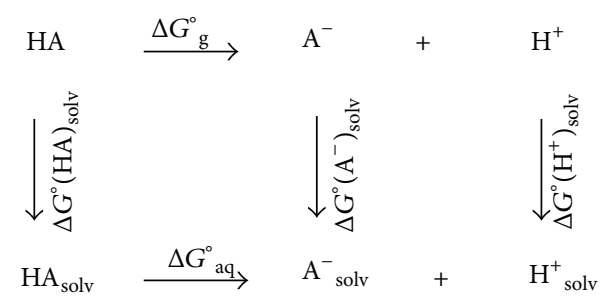

FIGURE 4: Thermodynamic cycle to compute $\mathrm{p} K_{\mathrm{a}}$ for an acid HA (see the text).

The CA molecule consists of specified p-electrons subsystems, the propenoic acid, and the catechol aromatic ring. The obtained spectrum most probably indicates that none of the observed bands are due to a transition characteristic of only one subsystem of the molecule. Hence, CA molecule consists mainly of one pi system and not of a number of independent $\mathrm{p}$ systems [16]. Similar observation has been reported for cinnamic acid [16]. However, charge migration during light absorption induces CT character of the longest wavelength transition as indicated in Table 1 and Figure 3. The MOs shown in Figure 3 is characterized by a number of nodes, indicating a disturbance of the pi system of CA molecule leading to limited CT character of the electronic transitions. The source of disturbance of the pi system is due to building up of opposing electric dipoles between the electron-withdrawing carboxylic group and the electrondonating vinyl group. This results in low intensity and high energy of the observed bands [16]. This is confirmed by the small value of the computed dipole moment in the $\mathrm{S}_{0}$ state (2.14 Debye), which is decreased in the $\mathrm{S}_{1}$ (1.02 Debye) state. This is also verified by the observed negative solvatochromism of CA [17] where its longest wavelength band is shifted to longer wavelength in nonpolar solvent due to stabilizing the $S_{1}$ relative to the $S_{0}$ state.

\subsection{Acidity Constant $\left(\mathrm{p} K_{\mathrm{a}}\right)$ Determination}

3.2.1. Experimental Determination of CA Acidity Constants $\left(\mathrm{p} K_{\mathrm{a}}\right)$. To determine the acidity constants of CA sets of absorption spectra were measured in different $\mathrm{pH}$ solutions, which are set to different values by means of sodium hydroxide and perchloric acid. Clear isosbestic points emerged by changing $\mathrm{pH}$ due to the equilibrium between the protonated and deprotonated CA species (see inset of Figure 2).

3.2.2. Computational Model for the $\mathrm{p} K_{\mathrm{a}}$ Calculation. Thermodynamic cycle depicted in Figure 4 shows the relation between the thermodynamic parameters of gas and aqueous phase. It can be calculated from (1)-(3) as follows [18]:

$$
\mathrm{p} K_{\mathrm{a}}=\frac{\Delta G_{(\mathrm{aq})}}{2.303 R T}
$$




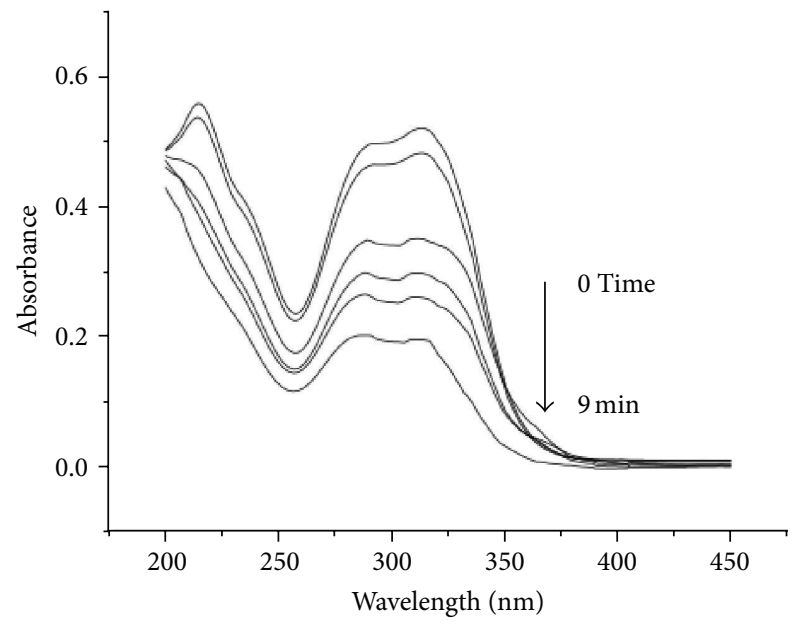

(a)

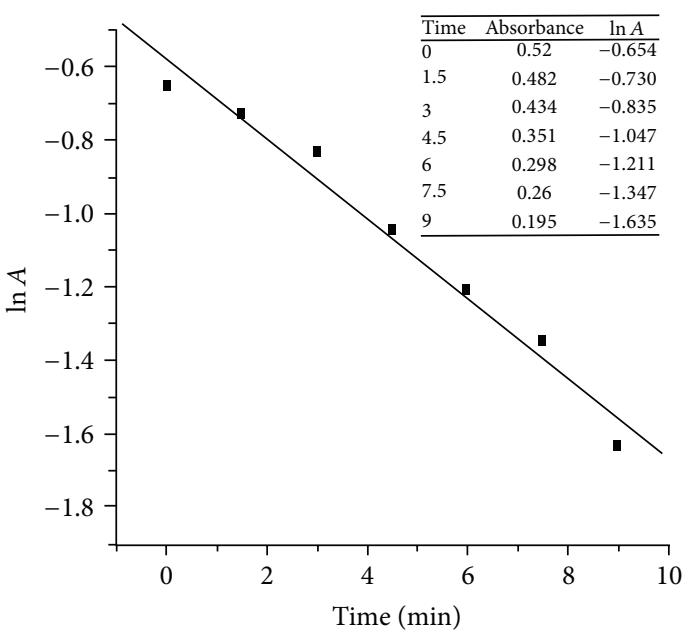

(b)

FIGURE 5: Effect of light irradiation time on the absorption spectrum of $\mathrm{CA}\left(0.6 \times 10^{-4} \mathrm{M}, \mathrm{pH}=3.3\right)$ in aqueous medium in presence of $\mathrm{TiO}_{2}$ $(0.5 \mathrm{~g} / \mathrm{L})(r=-0.996)$.

TABLE 2: Experimentally determined and theoretically calculated $\mathrm{p} K_{\mathrm{a}}$ values of CA.

\begin{tabular}{lccc}
\hline & This work & Literature values $^{*}$ & Theoretical value $^{* *}$ \\
\hline $\mathrm{p} K_{\mathrm{a} 1}$ & $4.5 \pm 0.1$ & $3.6-4.49$ & 4.05 \\
$\mathrm{p} K_{\mathrm{a} 2}$ & $8.6 \pm 0.2$ & $8.6-9.3$ & 6.27 \\
$\mathrm{p} K_{\mathrm{a} 3}$ & $10.3 \pm 0.1$ & $10.3-12.7$ & 10.23 \\
\hline
\end{tabular}

${ }^{*}$ See [12].

** Obtained from the quantum chemically calculated Gibbs free energies (Section 3.2.2).

which is a rearranged form of the famous thermodynamic relation $\Delta G=-R T \ln \left(K_{\mathrm{a}}\right)$ :

$$
\begin{aligned}
\Delta G_{(\mathrm{aq})}= & \Delta G_{\mathrm{g}}^{\circ}+\Delta G_{\text {solv }}^{\circ}\left(\mathrm{A}^{-}\right)+\Delta G_{\text {solv }}^{\circ}\left(\mathrm{H}^{+}\right) \\
& -\Delta G_{\text {solv }}^{\circ}(\mathrm{HA})+7.91\left(\mathrm{~kJ} \mathrm{~mol}^{-1}\right), \\
\Delta G_{\mathrm{g}}^{\circ}= & \Delta G_{\mathrm{g}}^{\circ}\left(\mathrm{A}^{-}\right)+\Delta G_{\mathrm{g}}^{\circ}\left(\mathrm{H}^{+}\right)-\Delta G_{\mathrm{g}}^{\circ}(\mathrm{HA}) .
\end{aligned}
$$

The quantity $7.91\left(\mathrm{~kJ} \mathrm{~mol}^{-1}\right)$ is the suggested correction value to coupe with standard free energy when changing the state from gas phase to aqueous [18]. Details of the methodology and used thermodynamic quantities are discussed in detail elsewhere [18]. Here we used the gas phase free energies $\Delta G_{\mathrm{g}}^{\circ}$ and the solvation free energies $\Delta G^{\circ}$ solv directly obtained from the computations. Using literature value of $\Delta G_{\mathrm{g}}^{\circ}\left(\mathrm{H}^{+}\right)$ and $\Delta G_{\text {solv }}^{\circ}\left(\mathrm{H}^{+}\right),-0.00711$ and -0.414 au, respectively, we were able to determine $\mathrm{p} K_{\mathrm{a}}$ values given in Table 2. Good prediction, at least in the trend, could be obtained. It is worth notifying that one should apply (2) and (3) taking into account the corresponding species generated step wisely from ionizing the tribasic $\mathrm{CA}\left(\mathrm{H}_{3} \mathrm{~A}\right)$ to $\mathrm{H}_{2} \mathrm{~A}^{-}, \mathrm{HA}^{2-}$, and $\mathrm{A}^{3-}$ species.
The values of Table 2 obtained theoretically correspond to the following stepwise dissociations:

$$
\begin{gathered}
\mathrm{pK}_{\mathrm{a} 1}: \mathrm{H}_{3} \mathrm{~A}_{\mathrm{aq}} \longrightarrow \mathrm{H}_{\mathrm{aq}}{ }^{+}+\mathrm{H}_{2} \mathrm{~A}_{\mathrm{aq}}{ }^{-} \\
\mathrm{pK}_{\mathrm{a} 2}: \mathrm{H}_{2} \mathrm{~A}_{\mathrm{aq}}{ }^{-} \longrightarrow \mathrm{H}_{\mathrm{aq}}{ }^{+}+\mathrm{HA}_{\mathrm{aq}}{ }^{2-} \\
\mathrm{pK}_{\mathrm{a} 3}: \mathrm{HA}_{\mathrm{aq}}{ }^{2-} \longrightarrow \mathrm{H}_{\mathrm{aq}}{ }^{+}+\mathrm{A}_{\mathrm{aq}}{ }^{3-}
\end{gathered}
$$

Table 3 contains the data used for $\mathrm{p} K_{\mathrm{a}}$ determination.

3.3. Photocatalytic Studies. CA solution was prepared at $\mathrm{pH}=$ 3.3. It continuously irradiated by xenon arc lamp at different time intervals. UV-Vis spectrum is monitored spectrophotometrically and depicted in Figure 5. The determined zeroorder rate constant equals $0.09 \mathrm{~min}^{-1}$ Figure 5. Change in Bond Order (Mulliken) upon irradiation is computed. Shadowed bonds in Figure 6 exhibit largest change in $\mathrm{BO}$ pointing to the most probable bond cleavage locations leading to photodegradation.

3.3.1. The Effect of Metal Ions. We observed similar behavior in presence of $\mathrm{Fe}^{3+}$ or $\mathrm{Cu}^{2+}$ chloride. However, in case of absence of metal ions and in presence of $\mathrm{Cu}^{2+}$ and $\mathrm{Fe}^{3+}$, the rate of degradation is lowered in the order $0.37>0.09>$ $0.05 \mathrm{~min}^{-1}$, respectively.

Consequently, iron ion is the most photostabilizer of CA. Most probably this is due to strong ground state complex formation that alters electronic structure and strengthens molecular bonds. Similar results based on both theoretical and experimental results of $\mathrm{Al}^{3+}$ ions with $\mathrm{CA}$ have been reported [19].

It is also worth pointing out that the presence of cations in the solution could act as electron scavenger leading to reduce photocatalytic efficiency of $\mathrm{TiO}_{2}$. When adding $\mathrm{Cu}^{2+}$ or $\mathrm{Fe}^{3+}$ the increase in the stability may be attributed to the 
TABLE 3: Gibbs free energies used for calculating $\mathrm{pK}_{\mathrm{al}-3}$ obtained in this work.

\begin{tabular}{lccccc}
\hline$\Delta G^{\circ}$ & $\mathrm{H}_{3} \mathrm{~A}$ & $\mathrm{H}_{2} \mathrm{~A}^{-}$ & $\mathrm{HA}^{2-}$ & $\mathrm{A}^{3-}$ & $\mathrm{H}^{+}$ \\
\hline Gas $^{*}$ & -648.5100 & -647.956533 & -647.28500 & -646.457122 & -0.00711 \\
Solvation $^{* *}$ & -70.7 & -329.5 & -812.5 & -1676.9 & -1087 \\
\hline
\end{tabular}

*a.u. units.

${ }^{* *} \mathrm{~kJ} \mathrm{~mol}^{-1}$.
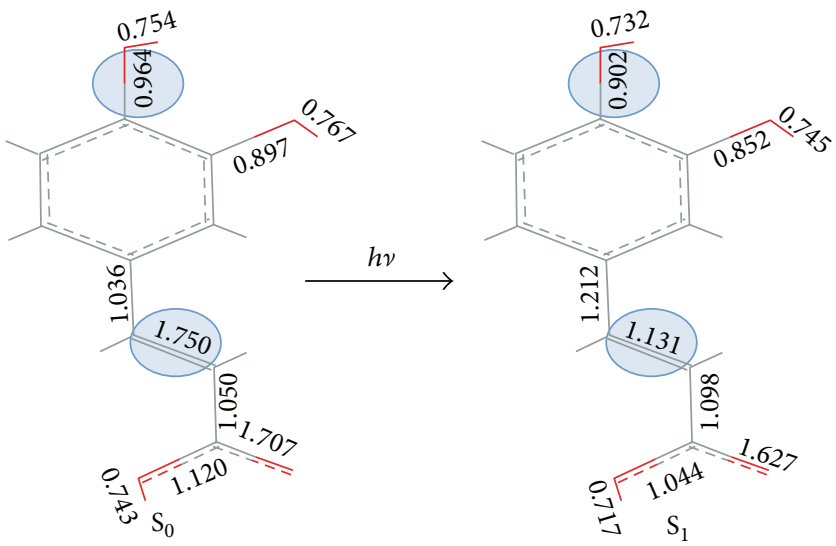

FIGURE 6: Change in Bond Order (Mulliken) upon irradiation. Shadowed bonds exhibit largest change in BO pointing to the most probable bond cleavage locations leading to photodegradation.

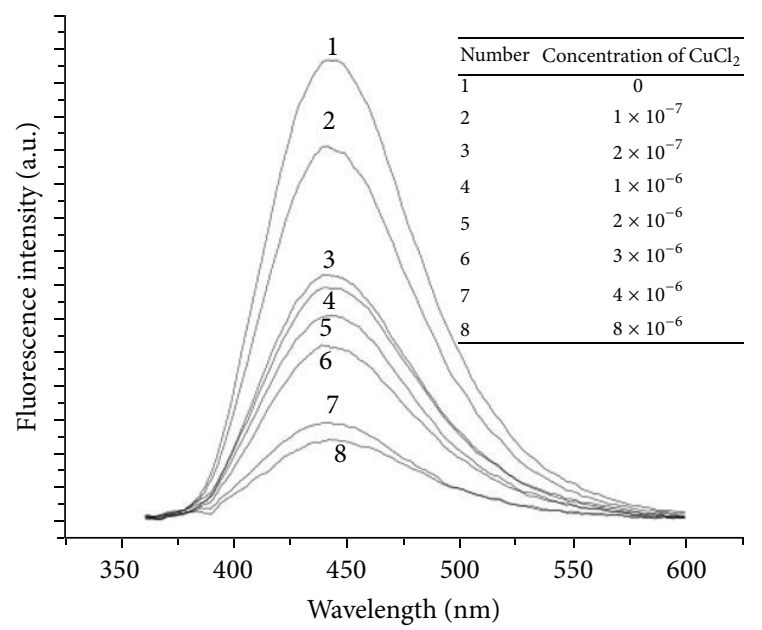

(a)

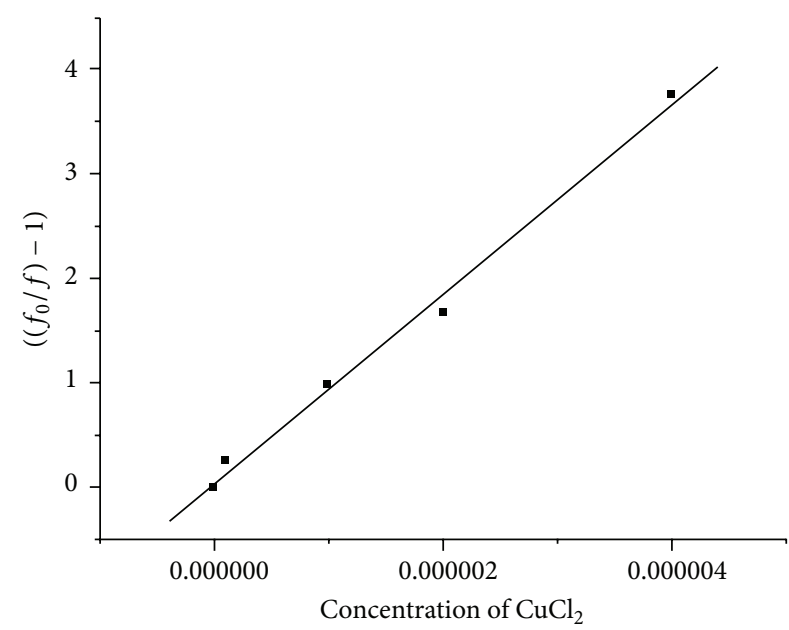

(b)

FIgURE 7: Emission spectra of CA $\left(1 \times 10^{-6} \mathrm{M}\right)$ in presence of various concentrations of $\mathrm{CuCl}_{2}$ and Stern-Volmer plot.

$\mathrm{Cu}^{+} / \mathrm{Cu}^{2+}$ or $\mathrm{Fe}^{2+} / \mathrm{Fe}^{3+}$ redox couple functions that shortcircuit the $\mathrm{TiO}_{2}$. Similar effects were reported for $\mathrm{Cu}^{2+}$ ions in photocatalytic decomposition of phenol by $\mathrm{TiO}_{2}[20]$.

The following section will show that fluorescence quenching of CA induced by addition of metal ions supports the suggestion of ground state complex formation as the main reason for the observed extra photostabilization by cations.

3.4. Metal Ions-Induced Fluorescence Quenching of CA. The dependence of the fluorescence as a function of the quencher concentration follows the Stern-Volmer relation: $F_{0} / F=$ $1+K_{\mathrm{sv}}[\mathrm{Q}]$, where $K_{\mathrm{sv}}$ is the Stern-Volmer quenching rate constant and its value represents the association constant of the complex $\left(K_{\mathrm{a}}\right)$.

The quenching of fluorescence of CA was studied by observing the changes in emission intensity in presence of $\mathrm{Fe}^{+3}$ or $\mathrm{Cu}^{+2}$ chloride. The fluorescence quenching experiments were carried out at different quencher concentrations. The complexes formed with both $\mathrm{Cu}^{+2}$ and $\mathrm{Fe}^{+3}$ ions are nofluorescence exciplexes since there is no shift appearing in the fluorescence band.

The variation of emission spectrum of $\mathrm{CA}\left(1 \times 10^{-6} \mathrm{M}\right)$ in presence of various concentrations of $\mathrm{CuCl}_{2}$ is represented in Figure 7 . The quenching rate constant $K_{\mathrm{sv}}\left(90.638 \mathrm{~kL} \cdot \mathrm{mol}^{-1}\right)$ 
TABLE 4: Frontier Orbital Energies and the determined reactivity descriptors [13]: chemical potential $\mu$, hardness ( $\eta$ ), and softness of CA molecule.

\begin{tabular}{|c|c|c|c|}
\hline MO energy $(\mathrm{eV})$ & $\mu=\left(\varepsilon_{\mathrm{HOMO}}+\varepsilon_{\mathrm{LUMO}}\right) / 2$ & $\eta=\varepsilon_{\mathrm{LUMO}}-\varepsilon_{\mathrm{HOMO}}$ & $S=1 / \eta$ \\
\hline $\begin{array}{l}\varepsilon_{\mathrm{HOMO}}=-5.84 \\
\varepsilon_{\mathrm{LUMO}}=-1.69\end{array}$ & -3.765 & 4.15 & 0.241 \\
\hline
\end{tabular}

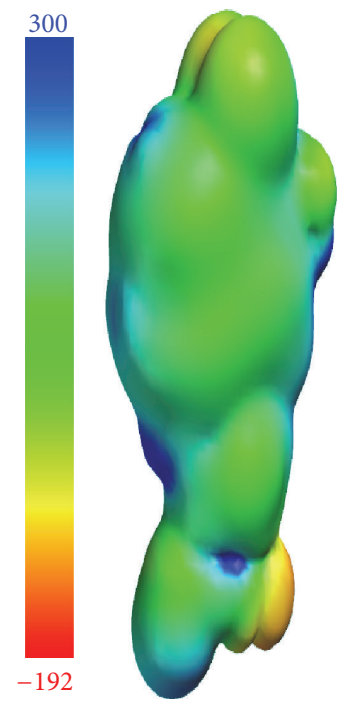

$\operatorname{EPS} \operatorname{map}\left(\mathrm{S}_{0}\right.$ state $)$
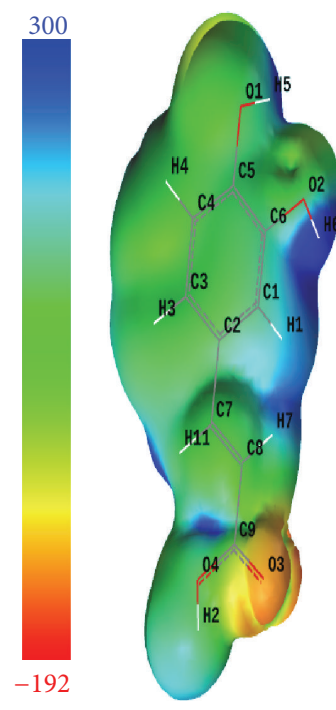

A cut-away of EPS map

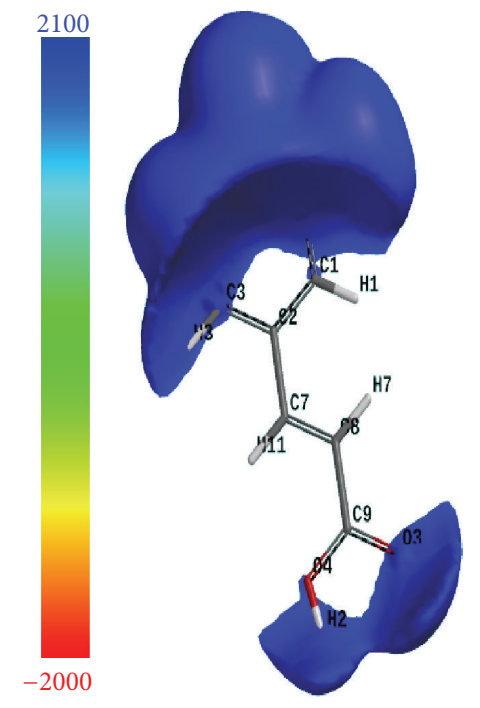

EPS map in the $S_{1}$ state

FIGURE 8: EPS maps of CA in $\mathrm{S}_{0}$ and $\mathrm{S}_{1}$ states showing PE range in $\mathrm{kJ} \mathrm{mol}^{-1}$ (EPS map giving the electrostatic potential at locations on total electron density surface corresponding to overall molecular size). Higher EPS in $S_{1}$ state with positive values at both molecular ends could be used as indicator for bond rupture at the $-\mathrm{C}_{7}=\mathrm{C}_{8}$ - group.

LUMO map (nucleophilic indicator)
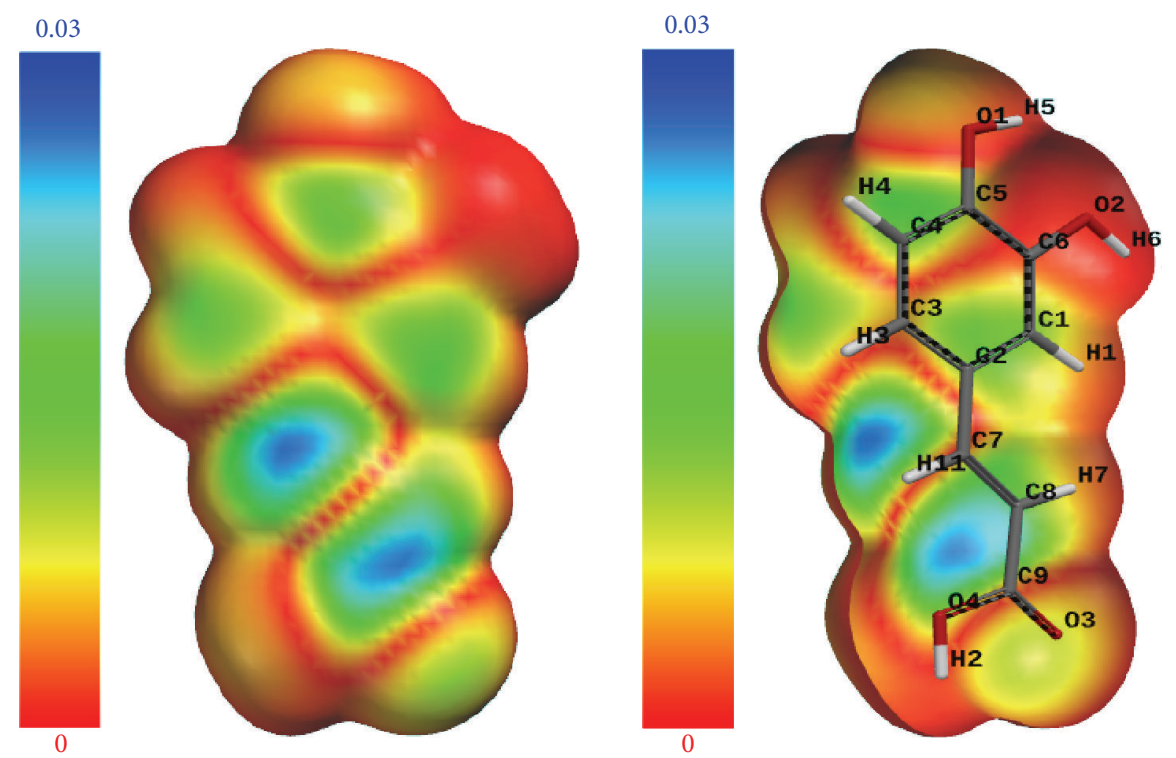

FIGURE 9: Nucleophilicity map. The LUMO map, wherein the absolute value of the LUMO is mapped onto the total SCF calculated electron density, providing an indication of nucleophilic reactivity (blue region is the highest reactive nucleophilic site). 

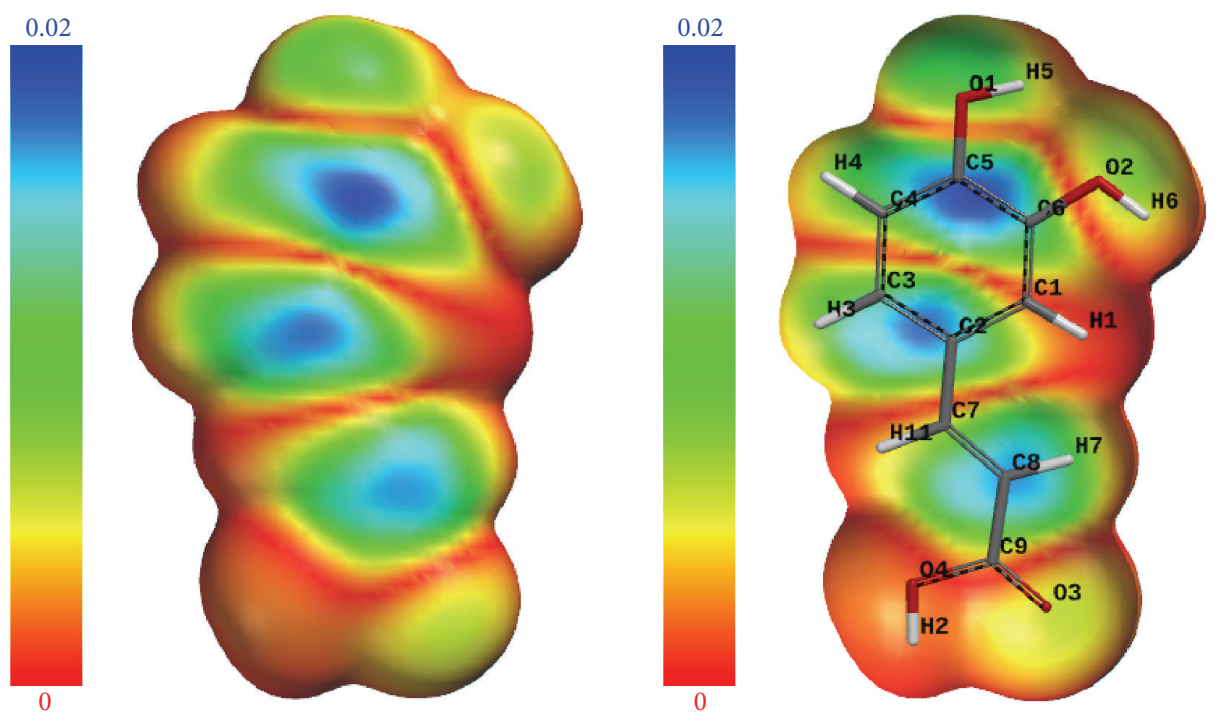

FIGURE 10: Electrophilicity map. The HOMO map, wherein the absolute value of the HOMO is mapped onto the total SCF calculated electron density, providing an indication of electrophilic reactivity (blue region is the highest reactive electrophilic site).
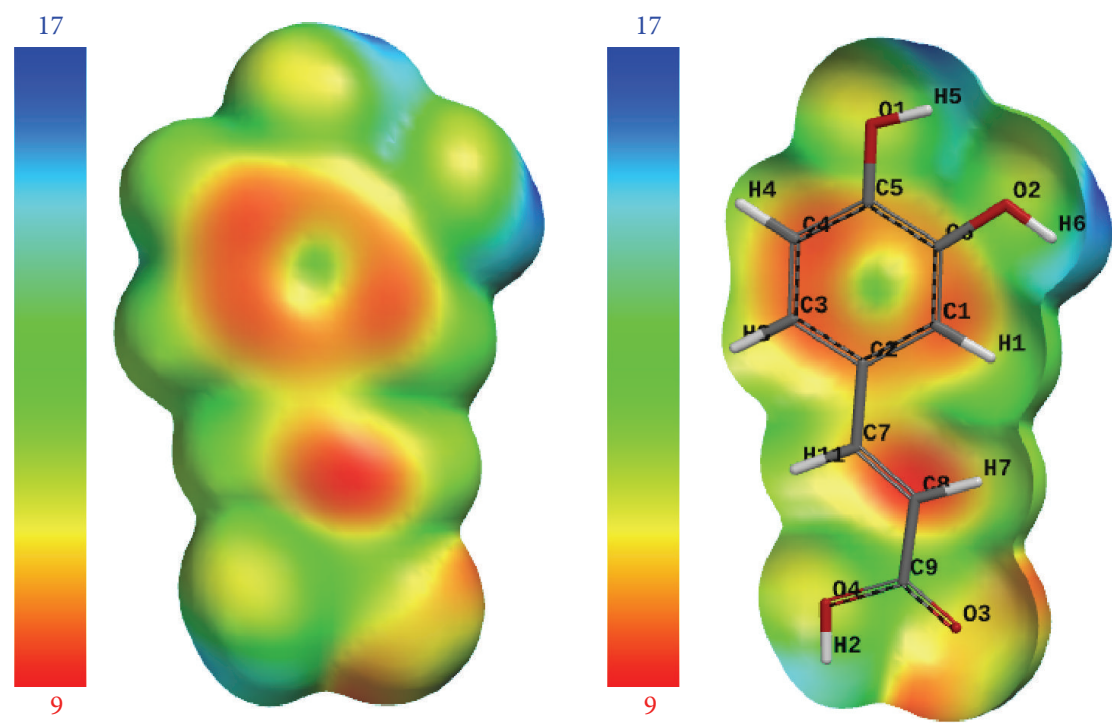

FIGURE 11: Ionization potential map. The local ionization potential map, defined as the sum over orbital electron densities, $\rho i(\mathbf{r})$ times absolute orbital energies, $\epsilon i$, and divided by the total electron density, $\rho(\mathbf{r})$. The local ionization potential reflects the relative ease of electron removal (ionization) at any location around CA molecule.

was determined from Stern-Volmer linear plot (with 0.996 correlation coefficient) between the fluorescence intensity of $\mathrm{CA}$ as a function of various concentration of $\mathrm{CuCl}_{2}$ in Figure 7.

Similar results were obtained for $\mathrm{FeCl}_{3}$ with much higher value of $K_{\mathrm{sv}}\left(363.625 \mathrm{~kL} \cdot \mathrm{mol}^{-1}\right)$ reflecting the enhanced stability of the excited state complexes of iron relative to copper. Similar effects were reported for $\mathrm{Cu}^{+2}$ ions in a study on the photocatalytic decomposition of phenol at the $\mathrm{TiO}_{2}$ [20].

3.5. Graphical Models and Molecular Reactivity [15, 21-23]. In order to visualize the structure-properties relationships we computed the electrostatic potential energy surface (ESP map), Figure 8, LUMO maps, Figure 9, HOMO maps, Figure 10, and ionization energy map, Figure 11. Moreover, reactivity descriptors such as chemical potential $(\mu)$, hardness $(\eta)$, and softness (S) of CA have been computed and tabulated in Table 4. Each of those surfaces and reactivity descriptors provides invaluable information about CA molecule such as its molecular shapes and electrostatic energy stability, its behavior towards nucleophiles, electrophiles, and its local ionization energy. These are presented in Figures 8-11, which are self-illustrative.

The chemical potential $\mu$ measures the escaping tendency of electron from equilibrium (could be considered as the 
negative value of the electronegativity of the molecule) and the global hardness $\eta$ can be seen as the resistance to charge transfer (or the band gap), while softness $S$ gives indication of how large is electron transfer to/from the molecule when the chemical potential changes [13].

\section{Conclusion}

The computed parameters that characterize molecular properties of CA are correlated with the experimental data. The origin of UV/Vis electronic transitions is assigned. The change in the computed bond orders during light irradiation sheds light on the photodegradation initial step.

Computed acidity constants satisfactorily correlated with experimentally determined $\mathrm{p} K_{\mathrm{a}}$ values.

Molecular graphical surfaces showed the maps of nucleophilicity, electrophilicity, ionization energies distribution, and the electrostatic potential energy maps. Chemical potential, hardness, and softness reactivity descriptors are also computed using HOMO-LUMO energies. These results give important information of the CA molecular reactivity.

The conclusions derived from this work can be very useful in the understanding of the chemical processes undergone by CA, which may contribute to a deeper understanding of the nutritional value of the foods where this molecule appears.

\section{Conflict of Interests}

The author declares that there is no conflict of interests regarding the publication of this paper.

\section{References}

[1] V. Marques and A. Farah, "Chlorogenic acids and related compounds in medicinal plants and infusions," Food Chemistry, vol. 113, no. 4, pp. 1370-1376, 2009.

[2] F. Natella, M. Nardini, I. Giannetti, C. Dattilo, and C. Scaccini, "Coffee drinking influences plasma antioxidant capacity in humans," Journal of Agricultural and Food Chemistry, vol. 50, no. 21, pp. 6211-6216, 2002.

[3] F. Natella, M. Nardini, F. Belelli, and C. Scaccini, "Coffee drinking induces incorporation of phenolic acids into LDL and increases the resistance of LDL to ex vivo oxidation in humans," American Journal of Clinical Nutrition, vol. 86, no. 3, pp. 604609, 2007.

[4] M. Nardini, F. Natella, V. Gentili, M. D. Felice, and C. Scaccini, "Effect of caffeic acid dietary supplementation on the antioxidant defense system in rat: an in vivo study," Archives of Biochemistry and Biophysics, vol. 342, no. 1, pp. 157-160, 1997.

[5] A. A. Johnson, C. Marchand, and Y. Pommier, "HIV-1 integrase inhibitors: a decade of research and two drugs in clinical trial," Current Topics in Medicinal Chemistry, vol. 4, no. 10, pp. 10591077, 2004.

[6] M. R. Fesen, Y. Pommier, F. Leteurtre, S. Hiroguchi, J. Yung, and K. W. Kohn, "Inhibition of HIV-1 integrase by flavones, Caffeic Acid Phenethyl Ester (CAPE) and related compounds," Biochemical Pharmacology, vol. 48, no. 3, pp. 595-608, 1994.

[7] M. Hirose, Y. Takesada, H. Tanaka, S. Tamano, T. Kato, and T. Shirai, "Carcinogenicity of antioxidants BHA, caffeic acid, sesamol, 4-methoxyphenol and catechol at low doses, either alone or in combination, and modulation of their effects in a rat medium-term multi-organ carcinogenesis model," Carcinogenesis, vol. 19, no. 1, pp. 207-212, 1998.

[8] M. Mir Derikvand, J. B. Sierra, K. Ruel et al., "Redirection of the phenylpropanoid pathway to feruloyl malate in Arabidopsis mutants deficient for cinnamoyl-CoA reductase 1," Planta, vol. 227, no. 5, pp. 943-956, 2008.

[9] L. Pari and A. Prasath, "Efficacy of caffeic acid in preventing nickel induced oxidative damage in liver of rats," ChemicoBiological Interactions, vol. 173, no. 2, pp. 77-83, 2008.

[10] D. Bassil, D. P. Makris, and P. Kefalas, "Oxidation of caffeic acid in the presence of L-cysteine: isolation of 2-S-cysteinylcaffeic acid and evaluation of its antioxidant properties," Food Research International, vol. 38, no. 4, pp. 395-402, 2005.

[11] M. Leopoldini, N. Russo, and M. Toscano, "The molecular basis of working mechanism of natural polyphenolic antioxidants," Food Chemistry, vol. 125, no. 2, pp. 288-306, 2011.

[12] M. Coimbra, B. Isacchi, L. Van Bloois et al., "Improving solubility and chemical stability of natural compounds for medicinal use by incorporation into liposomes," International Journal of Pharmaceutics, vol. 416, no. 2, pp. 433-442, 2011.

[13] F. Zielinski, V. Tognetti, and L. Joubert, "Condensed descriptors for reactivity: a methodological study," Chemical Physics Letters, vol. 527, pp. 67-72, 2012.

[14] M. J. Frisch, G. W. Trucks, H. B. Schlegel et al., GAUSSIAN 09, Revision D.01, Gaussian, Wallingford, Conn, USA, 2014.

[15] W. J. Hehre, A. Shusterman, and J. Nelson, Molecular Modeling Workbook for Organic Chemistry, Wavefunction, Inc, 1998.

[16] R. H. Abu-Eittah, M. K. Khedr, M. Goma, and W. Zordok, "The structure of cinnamic acid and cinnamoyl azides, a unique localized $\pi$ system: the electronic spectra and DFT-treatment," International Journal of Quantum Chemistry, vol. 112, no. 5, pp. 1256-1272, 2012.

[17] H. R. Galal, Light induced reactions on $\mathrm{TiO}_{2}$ surfaces [M.S. thesis], Faculty of Science, Ain Shams University, 2000.

[18] C. P. Kelly, C. J. Cramer, and D. G. Truhlar, "Adding explicit solvent molecules to continuum solvent calculations for the calculation of aqueous acid dissociation constants," Journal of Physical Chemistry A, vol. 110, no. 7, pp. 2493-2499, 2006.

[19] C. Lapouge and J.-P. Cornard, "Reaction pathways involved in the mechanism of AlIII chelation with caffeic acid: catechol and carboxylic functions competition," ChemPhysChem, vol. 8, no. 3, pp. 473-479, 2007.

[20] K. Rajeshwar and J. G. Ibanez, "Electrochemical aspects of photocatalysis: application to detoxification and disinfection scenarios," Journal of Chemical Education, vol. 72, no. 11, pp. 1044-1049, 1995.

[21] A. J. Shusterman and G. P. Shusterman, "Teaching chemistry with electron density models," The Journal of Chemical Education, vol. 74, no. 7, pp. 771-776, 1997.

[22] M. B. Smith, Organic Synthesis, Wavefunction Inc, 3rd edition, 2010.

[23] K. J. Linenberger, R. S. Cole, and S. Sarkar, "Looking beyond Lewis structures: a general chemistry molecular modeling experiment focusing on physical properties and geometry," The Journal of Chemical Education, vol. 88, no. 7, pp. 962-965, 2011. 

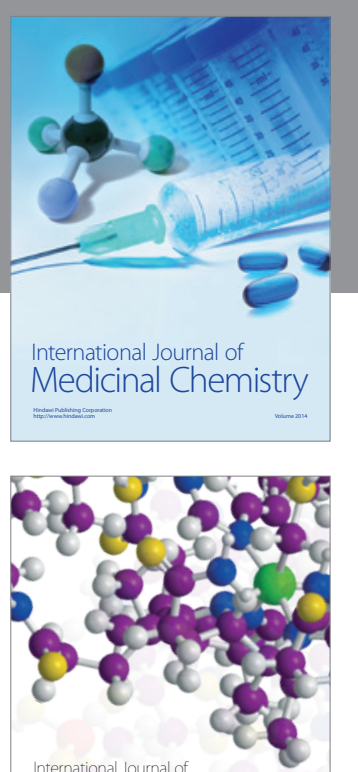

Carbohydrate Chemistry

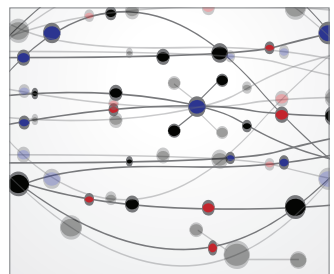

The Scientific World Journal
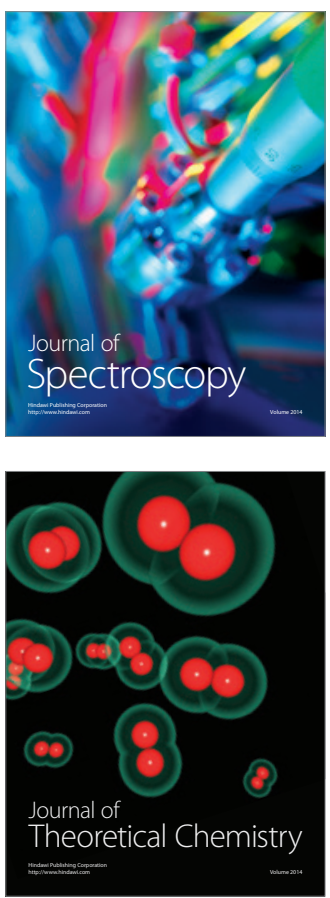
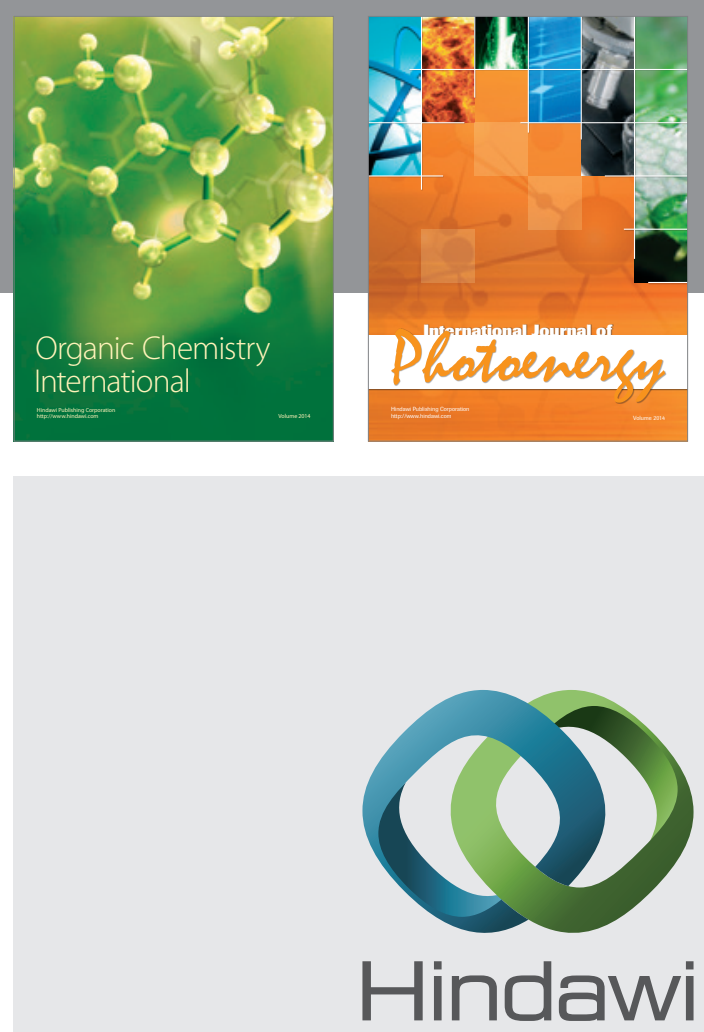

Submit your manuscripts at

http://www.hindawi.com

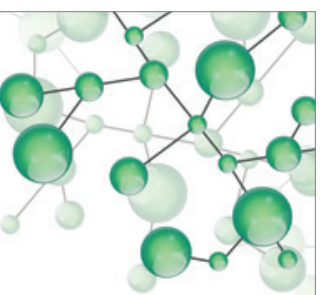

International Journal of

Inorganic Chemistry

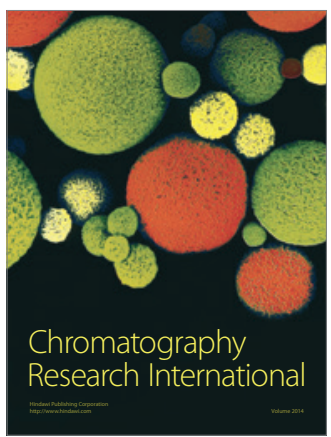

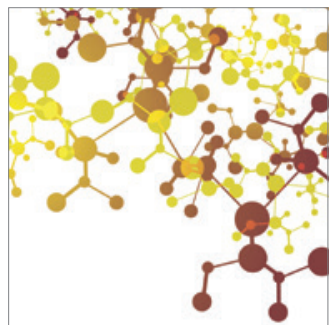

Applied Chemistry
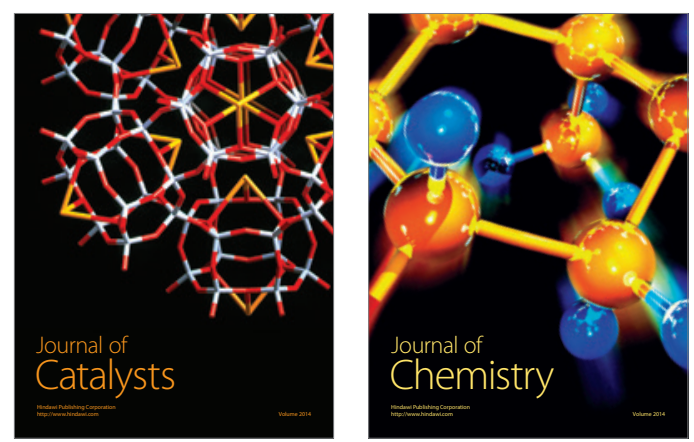
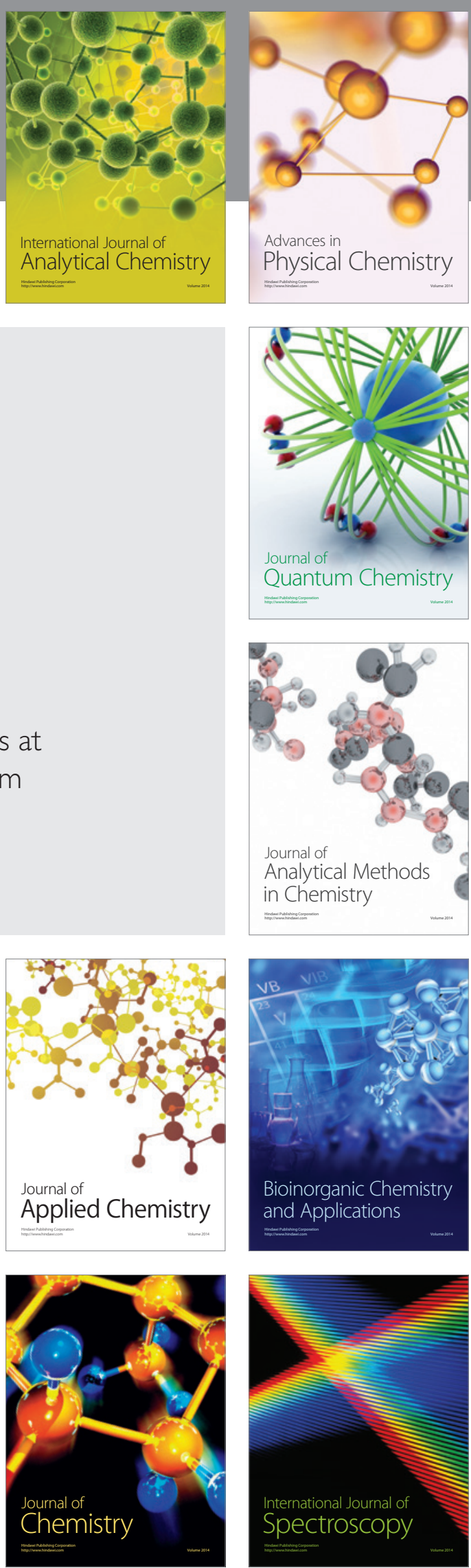